\title{
Screening for Diabetic Retinopathy in Communities
}

\author{
CATHIE J. STEPIEN, RN, MPH; MARILYN A. BOWBEER; ROLAND G. HISS, MD \\ The University of Michigan Diabetes Research and Training Center \\ Continuing Education and Outreach Core \\ Ann Arbor, Michigan
}

Twelve retinopathy screening clinics serving 489 diabetic patients were conducted in three Michigan communities as part of the outreach effort of the Michigan Diabetes Research and Training Center: Screening activities were initiated by local diabetes educators who conducted a program designed to promote detection of diabetic eye disease and increase patient and health care provider awareness of accepted ophthalmic evaluation guidelines. This experience suggests that retinopathy screening clinics can be successfully conducted if health care professionals in the community consider diabetic retinopathy to be a serious problem, one individual is willing to oversee the organizational aspects of the clinic, and an ophthalmologist with laser treatment capability is present or nearby. These clinics are effective in detecting diabetic eye disease and facilitating subsequent patient visits to an ophthalmologist for evaluation in accordance with national recommendations.
A decade ago, proliferative retinopathy and other severe diabetic eye complications frequently led to blindness or severely impaired vision. Today, laser treatment and vitrectomy are available to treat those disorders. The Diabetic Retinopathy Study (DRS) demonstrated that laser treatment initiated in a timely fashion is effective in preserving vision and ameliorating the severe effects of diabetic retinal disease. ' Because serious diabetic retinopathy is often asymptomatic and its detection difficult. several recommendations have been made by the National Diabetes Advisory Board (NDAB) for routine ophthalmologic examination to ensure timely detection and treatment. The critical importance of identification and referral for treatment was emphasized by the NDAB in its National Plan to Reduce Mortality and Morbidity of Diabetes. ${ }^{2}$ Further, there was a substantial effort to make practicing physicians aware of the DRS Group's findings. In 1983, the NDAB published and disseminated a booklet entitled The Prevention and Treatment of Five Complications of Diabetes: A Guide for Primary Care Practitioners. ${ }^{3}$ This booklet specifically addresses the prevention, detection/monitoring, and treatment/referral of diabetic eye disease. In Michigan, the Department of Public Health in 1984 developed and distributed diabetic retinopathy referral guidelines for health care providers throughout the state. ${ }^{+}$

Despite these activities, there was strong evidence that the guidelines were not being followed at the community level. A study by Stross and Harlan ${ }^{5}$ reported that 18 months after the DRS findings were reported, $72 \%$ of family physicians and $54 \%$ of internists were not aware of the study and its implications for referral and treatment. A survey conducted in Michigan a year following the dissemination of the retinopathy referral guidelines found that many health professionals who had received the guidelines had not read them. Among diabetic individuals surveyed, only $26 \%$ reported that they had been given the complete recommendations for eye care delineated in the guidelines. ${ }^{6}$ In the 1985 assessment of the status of diabetes care in Michigan, the review of the data revealed that, among 261 patients from eight communities, only $59 \%$ of patients with diabetes had seen an

This work was supported by National Institutes of Health Grant \#NIH 2 P60 DK20572, National Institute of Diabetes Digestive and Kidney Diseases, Bethesda, Maryland.

Reprint requests to Cathie J. Stepien, RN, MPH, The University of Michigan, Department of Postgraduate Medicine, Towsley Center, Ann Arbor, Ml 48109-0201. 
ophthalmologist at some time and only $54 \%$ had seen one within the last 2 years. ${ }^{?}$

In an effort to address this problem, screening for retinopathy has been undertaken by a number of groups to detect previously unrecognized disease and to highlight the importance of annual ophthalmologic evaluation. These studies have been conducted using a mobile examination van and/or personnel who perform fundus photography in the field, with subsequent evaluation of the photographs by an ophthalmologist. $^{8-10}$ This format has been efficacious in identifying cases of diabetic eye disease and has other benefits as well; screening activities that use a mobile examination van allow evaluation of large numbers of people in geographically dispersed areas, and the use of fundus photography helps to resolve the issue of labor-intensive screening by health professionals whose services may be difficult to acquire on a volunteer basis. However, many communities lack the financial resources to purchase such equipment and may wish to consider other alternatives.

The retinopathy screening experience described in this report provides another model for conducting screening at the community level. This paper will describe the components of a community-based retinopathy screening clinic, the role of the diabetes educator to initiate the project and mobilize the necessary resources, and the significant patient findings that demonstrate the value of such an undertaking.

\section{Research Design and Methods}

As part of the outreach effort of the Michigan Diabetes Research and Training Center (MDRTC), diabetes advisory councils (DACs) have been developed in each of six communities randomly selected within the state of Michigan. Each council has representatives from the local diabetes community, including health professionals and consumers interested in diabetes. Council members, in collaboration with the staff of the MDRTC, study the diabetes care in their own communities, noting problems and trends in diabetes care and addressing them through the development of programs at the community level. Three DACs were particularly concerned that the national recommendations regarding ophthalmologic care for persons with diabetes were not being met. To promote detection of ophthalmologic disease among people with diabetes, 12 retinopathy screening clinics serving 489 diabetic patients were conducted in these three communities over the course of 2 years.

In each community, a diabetes educator from a local hospital assumed responsibility for organizing the clinic. Space was provided by the hospital or a community ophthalmologist, and the diabetes educator handled the advance publicity. To announce the availability of the clinics, diabetes educators developed fliers that were mailed to and posted in primary care physicians' offices. Each physician also received a letter that detailed the diabetic retinopathy referral guidelines and encouraged the physician to follow the guidelines, if not already doing so. Notices were placed in the newspaper, public service announcements were made on radio and television; and posters were displayed in various locations such as pharmacies, clinics, and other community locations. In some instances, personal mailings were made to members of diabetes patient support groups. Not all publicity measures were used in each community.
The local chapter of the American Diabetes Association or the diabetes educator scheduled the patient appointments and assembled the necessary volunteer assistance from among local health professionals. Volunteers generally included (1) someone to check the patients in and coordinate patient flow through all stations, (2) interviewers to review the medical and diabetes history questionnaire with each patient, (3) a nurse or technician to obtain random blood glucose and physical assessment measures, (4) a nurse or technician to conduct visual acuity assessments and instill eye drops, and (5) the ophthalmologist and a person to assist the ophthalmologist in conducting the fundus examination and tonometry.

Ophthalmologist recruitment was conducted by advisory council members. Council members selected the ophthalmologist currently providing the most diabetes ophthalmologic care to the people of that community. These ophthalmologists were usually well known to the medical community and had laser treatment capabilities in their practices. The ophthalmologists were invited to volunteer their time to provide ophthalmologic exams at the screening clinics and were willing to participate.

The number of patients scheduled varied by community according to the number of volunteers and physical space available. In one community, a volunteer staff of eight persons (excluding the ophthalmologist) was assembled, the clinic was held in the office suite of the community's only ophthalmologist, and 1 patient could be scheduled every 5 minutes. In another community, only five volunteers were obtained; the clinic occupied a portion of the hospital corridor and two treatment rooms usually reserved for the Emergency Department. Because of the limited space, 1 patient could be scheduled every 10 minutes. In another community, the clinic was held in the education corridor of one of the community hospitals, occupying a suite of offices and conference rooms. Here 1 patient was scheduled every $5 \mathrm{~min}-$ utes. Generally, the ophthalmologists could comfortably screen 12 patients per hour. Fewer patients were scheduled if space and volunteer restrictions necessitated such a decision.

An advance mailing, which included a confirmation of the appointment and an interview questionnaire, was sent to each patient. A 52-item medical history questionnaire was used. Patients were asked to complete the questionnaire and bring it with them to the clinic. Height, weight, blood pressure, heart rate, and blood glucose values were determined. Blood for cholesterol, triglyceride, serum creatinine, C-peptide, and $\mathrm{HbA}_{1}$ measurement was drawn and taken back to the laboratories of the MDRTC to be analyzed. Visual acuity and intraocular pressure were assessed. Each patient received a complete funduscopic examination by the ophthalmologist. It should be noted that some of these steps and blood tests were conducted by the MDRTC staff for research purposes and are not integral to replication in all communities.

Those persons with ophthalmologic findings requiring further attention were instructed to seek an appointment for further evaluation with an area ophthalmologist within a specified time period. Those without serious findings were advised to have their eyes checked on an annual basis. The importance of annual ophthalmologic evaluation was reinforced. Each patient and his or her physician were sent the 
results of the ophthalmologic examination and the blood analysis.

To evaluate the effectiveness of the clinic, all patients with serious findings at any of the clinics were contacted by phone. These patients had been seen at screening clinics from 6 months to 3 years prior to the telephone follow-up. All others (ie, those without serious findings) were contacted by mail to determine their follow-through with the recommendation that they seek an annual ophthalmologic exam. Patients seen within the previous year, with either less serious findings or normal exams, were not contacted because their "annual" ophthalmologic exam would not be due yet.

\section{Results}

Clinic Activity and Data Set Overall, there were 552 visits to the screening clinics. Two communities each conducted five clinics consisting of 415 screening visits. However, both of those communities experienced significant repeat rates $(17 \%$ and $14 \%)$. In the other community, efforts were made to limit patients to one screening visit. They conducted two clinics, screening a total of 137 patients. The data set consists of 489 patients; repeat visits were not included. Also, the communities used different age-eligibility criteria (ie, one community allowed children to be screened, one allowed adolescents, and one required that the patients be adults). For purposes of uniformity, patients under 21 years of age were eliminated from the data set.

Effectiveness of Publicity Measures Extensive publicity measures were employed in each community, although not all communities used identical methods. To determine which publicity methods were most effective, patients were asked, "How did you first hear about the clinic?" Their responses are displayed in Table 1.

To ascertain what motivated the patients to attend the clinic, they were asked, "What was the most important reason you came to the clinic today?" The responses to this question are displayed in Table 2.

Patient Findings Table 3 displays the characteristics of the patients seen in the screening clinics.

Demographics Insulin-dependent diabetes patients were defined as insulin using. younger than age 40 at diagnosis of diabetes, less than $135 \%$ of ideal body weight, and having a random C-peptide value of $<0.6 \mathrm{ng} / \mathrm{mL}$. In terms of the racial distribution, $96 \%$ of patients screened were white and $2 \%$ were black. Although this distribution is not representative of the general population in Michigan, it reflects the population distribution of small, rural communities where the majority of screening took place. Attempts were made to provide working individuals ample opportunity to attend the screening clinics by holding some screenings on Saturdays and during evening hours.

Ophthalmologic Findings Forty-three percent of all patients had seen an ophthalmologist at some time in their life to check on the effects of diabetes on their eyes; $39 \%$ had seen an ophthalmologist in the past 2 years. Only $26 \%$ had ever been referred to an ophthalmologist by their physician. Among the $61 \%$ of patients who hadn't been to an ophthal-
Table 1. Effectiveness of Publicity Measures*

$\begin{array}{lc}\text { Publicity Methods } & \text { Responses } \\ \text { Newspaper } & 162(33) \\ \text { My physician told me } & 105(22) \\ \text { My diabetes educator told me } & 67(14) \\ \text { Support group/friends/family } & 50(10) \\ \text { Flyer or poster } & 29(6) \\ \text { Radio or TV ads } & 25(5) \\ \text { Personal mailing } & 20(4) \\ \text { Other } & 29(6)\end{array}$

* Patient response to the question, "How did you first hear about the clinic?"

Table 2. Motivation for Attending a Clinic*

\section{Reason for Attending}

To see if diabetes was affecting my eyes

Because I was having trouble seeing

$298(62)$

My doctor told me to

$46(10)$

My diabetes educator told me to

$30(6)$

It was a free clinic

$22(5)$

$21(4)$

I have never been to an ophthalmologist

$18(4)$

I' $m$ afraid I'll become blind

$13(3)$

Other

$32(6)$

* Patient responses to the question: "What was the most important reason you came to the clinic today?"

mologist in the past 2 years, the largest number (43\%) responded that they were not sent by their physician. An additional $20 \%$ did not feel it was important, and $41 \%$ didn't know they were supposed to consult an ophthalmologist. Only $16 \%$ reported cost as a reason for not seeking ophthalmologic evaluation. (Note: More than one answer was possible; hence, percentages add up to more than 100.)

Ophthalmologic abnormalities were identified in $50 \%$ of the people seen: $58 \%$ of those with insulin-dependent diabetes mellitus (IDDM) and $48 \%$ of those with non-insulindependent diabetes mellitus (NIDDM) had ophthalmologic findings. Normal retinas were found in 246 patients $(50 \%)$. Proliferative or preproliferative retinopathy was found in 11 $(2 \%)$ of those seen, macular edema in 6 patients $(1 \%)$, vitreous hemorrhage in 3 patients $(<1 \%)$, background retinopathy in 140 patients $(29 \%)$, cataracts in 66 patients $(13 \%)$, and other findings, including increased intraocular pressure, pigment changes, age-related degeneration, retinal changes of high blood pressure, and "other" were seen in $15 \%$ of the patients. (Percentages add up to more than 100 because some patients had more than one finding.) Ophthalmologic findings are displayed in Table 4.

Twenty patients $(4 \%)$ were identified as having serious findings. These findings included proliferative and preproliferative retinopathy (11 patients), vitreous hemorrhage (3 patients), and macular edema (6 patients). Among these 20 patients, only 6 reported that they knew diabetes had affected their eyes. Ten of these patients reported that they 


\begin{tabular}{|c|c|c|c|}
\hline Characteristics & $\begin{array}{c}\text { IDDM } \\
n=64(13 \%)\end{array}$ & $\begin{array}{c}\text { NIDDM } \\
\text { (Insulin Using) } \\
n=182(37 \%)\end{array}$ & $\begin{array}{c}\text { NIDDM } \\
\text { (Not Insulin Using) } \\
\mathbf{n = 2 4 3 ( 5 0 \% )}\end{array}$ \\
\hline Mean age & 40 & 63 & 64 \\
\hline \multicolumn{4}{|l|}{ Race } \\
\hline White & $61(95)$ & $174(96)$ & $238(98)$ \\
\hline Black & $1(2)$ & $8(4)$ & $4(2)$ \\
\hline Other & $2(3)$ & $\mathbf{0}$ & $1(<1)$ \\
\hline \multicolumn{4}{|l|}{ Gender } \\
\hline Male & $31(48)$ & $55(30)$ & $101(42)$ \\
\hline Female & $33(52)$ & $127(70)$ & $142(58)$ \\
\hline \multicolumn{4}{|l|}{ Duration of disease } \\
\hline Mean no. of years & 18 & 11 & 7 \\
\hline Mean $\mathrm{HbA}$, value $(\%)$ & 9.23 & 9.07 & 7.7 \\
\hline Practices SMBG† & $52(81)$ & $122(67)$ & $86(35)$ \\
\hline Had formal diabetes education & $52(81)$ & $133(73)$ & $102(42)$ \\
\hline
\end{tabular}

\begin{tabular}{|c|c|c|c|c|}
\hline Ophthalmologic Finding & $\begin{array}{c}\text { IDDM } \\
n=64\end{array}$ & $\begin{array}{c}\text { NIDDM } \\
\text { (Insulin Using) } \\
n=182\end{array}$ & $\begin{array}{c}\text { NIDDM } \\
\text { Not Insulin Using) } \\
\mathbf{n = 2 4 3}\end{array}$ & $\begin{array}{c}\text { Total } \\
n=489\end{array}$ \\
\hline Normal & $27(42)$ & $85(47)$ & $134(55)$ & $246(50)$ \\
\hline Background diabetic retinopathy & $30(47)$ & $57(3 \mathrm{l})$ & $53(22)$ & $140(29)$ \\
\hline $\begin{array}{l}\text { Proliferative or preproliferative } \\
\text { retinopathy }\end{array}$ & $5(8)$ & $5(2)$ & $1(<1)$ & $11(2)$ \\
\hline Vitreous hemorrhage & 0 & $1(<1)$ & $2(<1)$ & $3(<1)$ \\
\hline Macular edema & $1(2)$ & $2(1)$ & $3(1)$ & $6(1)$ \\
\hline Cataracts & $5(8)$ & $27(15)$ & $34(14)$ & $66(13)$ \\
\hline Increased intraocular pressure & 0 & $10(5)$ & $12(5)$ & $22(4)$ \\
\hline Pigment changes & $2(3)$ & $3(2)$ & $13(5)$ & $18(4)$ \\
\hline Age-related degeneration & 0 & $5(3)$ & $14(6)$ & $19(4)$ \\
\hline $\begin{array}{l}\text { Retinal changes of high blood } \\
\text { pressure }\end{array}$ & 0 & $2(1)$ & $1(<1)$ & $3(<1)$ \\
\hline Other & 0 & $6(3)$ & $4(2)$ & $10(2)$ \\
\hline
\end{tabular}

had seen an ophthalmologist within the last 2 years, and five reported that they had never seen an ophthalmologist before their attendance at this clinic.

Follow-up Each patient and his or her physician were sent the results of the ophthalmologic examination and the blood analysis. One hundred fifty-three physicians received reports on their patients.

Patients with serious findings at any of the clinics were contacted by phone to determine their follow-through with recommendations to seek immediate treatment. Telephone follow-up occurred on two occasions from 6 months to 3 years following the clinic visit. Fourteen of the 20 patients with serious findings were successfully contacted. Among these 14 patients, 1 had not returned to the ophthalmologist because of extensive illness; 1 did not see the ophthalmologist as recommended, but subsequently did receive treatment for retinopathy; and the other 11 did see an ophthalmologist as recommended. Of those 11 patients, 1 has become blind since the clinic, 5 have had surgery and/or laser photocoagulation, and 5 are being closely monitored. Among the 6 patients not contacted by telephone, 3 were seen at subsequent clinics. They continue to be monitored in the clinic setting. Three patients were lost to the study. They could not be located at the address and phone number they had provided, and other attempts to locate them were unsuccessful.

Follow-up postcards were sent to those patients who had been screened at least 1 year ago and who did not have serious findings. The postcard asked the patients how many times they had been to an ophthalmologist since attending 
the Diabetes Eye Clinic and if they had had laser treatments since attending the Diabetes Eye Clinic. Postcards were sent to 351 people and were retumed by 258 people for a response rate of $74 \%$. Of those who responded, $70 \%$ had returned to see an ophthalmologist at least once since the clinic.

\section{Discussion}

Twelve retinopathy screening clinics were successfully organized and implemented under the direction of local diabetes educators. Through effective publicity measures, full clinic schedules were easily generated in each community. The newspaper, the physician, and the diabetes educator were the most commonly mentioned sources of information about the clinics. Physician notification of the availability of the screening clinics served as an effective publicity measure for patients and had the secondary effect of disseminating ophthalmologic referral guidelines to the physician community. Over the course of the project, 153 physicians received follow-up information regarding the status of their patients seen at the retinopathy clinics.

The majority of patients $(62 \%)$ attended the clinic to see if diabetes was affecting their eyes. The screening clinics made it possible for diabetes educators to provide on-site patient teaching, not only about the importance of annual ophthalmologic evaluation, but about other areas of diabetes management as well. Many patients (41\%) had never participated in available diabetes education programs; the clinics fostered patient awareness of these valuable community resources. Aside from providing the opportunity to recruit patients to diabetes education in the community, these screening clinics also enabled diabetes educators to establish a more extensive mailing list for future activities and permitted the distribution of pertinent patient education materials. Further, obtaining other health information such as blood pressure and lipid measurements alerted patients, the diabetes educator, and the physician to other potential health concerns.

Ophthalmologic abnormalities were identified in $50 \%$ of those attending the screening clinics. Half of the patients (50\%) had normal retinas, and background retinopathy was detected among $28 \%$ of patients. Severe disease was found in $4 \%$ of those screened and, among those 20 patients, 5 reported that they had never seen an ophthalmologist to evaluate the effect of diabetes on their eyes. Thus, screening clinics may not uncover large quantities of undetected disease and may, in fact, serve as "interim visits" to those already under ophthalmologic care for serious conditions. Nevertheless, it was the experience in each screening clinic that less than half of the patients had ever seen an ophthalmologist. Even fewer (26\%) reported being referred to an ophthalmologist by their physician. Their attendance at the screening clinic provided an opportunity to learn about the possible effects of diabetes on the eyes, the availability of effective treatment measures for detected disease, and the importance of monitoring for these effects on a yearly basis.

Follow-up with clinic participants was conducted to ascertain the effectiveness of this activity in promoting adherence to the recommendation of annual ophthalmologic evaluation. Seventy percent of those responding to our postcard follow-up indicated that they had returned to the oph- thalmologist at least once since their clinic attendance. Prior to the screening clinic, $51 \%$ of these individuals had never been examined by an ophthalmologist.

From the positive experience in generating health professional and patient interest in a screening program and the significant educational rewards that are gained from it, it is reasonable to conclude that community-based retinopathy screening is a worthwhile endeavor. However, several themes emerged from the experience in conducting screening clinics in communities of different sizes. It is apparent that certain conditions within the professional community must be in place in order to conduct such clinics successfully. These characteristics include:

1. The health care professionals in the community must consider diabetic retinopathy to be a serious problem. Members of the Diabetes Advisory Councils in each of the communities were given data on the status of ophthalmologic care as assessed during our study of physicians and patients during the 1981-1985 period. Referral pattems of primary care physicians, patients' self-reports of frequency of visits to an ophthalmologist, purpose of the visit(s), and reasons for seeking or not seeking routine ophthalmologic evaluation were presented. Overall, barely half of all patients with NIDDM had ever seen an ophthalmologist and even fewer had received ophthalmologic evaluation within the past 2 years. Further, many patients indicated that they were not aware of the need to receive such an evaluation annually; many patients confused optometrists with ophthalmologists, and many reported that their physicians had not informed them of the need for this evaluation.

After reviewing the data, council members in several communities voiced an interest in conducting screening clinics. They expressed concern not only about unrecognized disease in their communities but also about patient and professional unawareness of the accepted guidelines for routine ophthalmologic evaluation. In these communities, council members were in agreement about the severity of the problem, and planning for screening activities was undertaken. In communities where council members did not find the data to be of great concern, or their perceptions of the potential difficulties in doing a screening program outweighed their interest in the problem, screening did not take place.

2. There must be one individual willing to coordinate the organizational aspects of the clinic. An essential component was the presence of one individual willing to oversee organizational issues. In all participating communities, the local diabetes educator assumed this role. Despite significant DRTC assistance with preparation of correspondence to patients and physicians, development of interview and examination forms, and so forth, the diabetes educator took the lead in securing the facility for screening, arranging volunteer assistance, and attending to the myriad of details that accompanied a project of this nature. In small communities with only one hospital and usually one educator, selection of the coordinator was not a problem. In large communities with several competing hospitals, however, there were conflicts involved in having a hospital-employed diabetes educator assume the responsibilities of organizing a "community" project. Although the activity was part of the educator's role as a Diabetes Advisory Council member, the educator was viewed as a representative of a particular hos- 
pital. For convenience, that hospital was also chosen as the site for conducting the screening. It therefore appeared to the community that the screening clinic was a function of the hospital and this became a source of tension among the health care professionals in the area. This was generally resolved by planning to rotate screening sites and coordinators. Regardless of community size, however, it was necessary that someone within that community take responsibility for planning.

3. An ophthalmologist who has laser treatment capability must be present or nearby. We learned that a particular community size or the presence of an ophthalmologist within that community is not necessary for a successful screening program. However, if a community has an ophthalmologist, he or she must have laser treatment capabilities in the office and be willing to participate in the project. Short of this, a willing ophthalmologist with laser equipment must be found elsewhere.

In one community, optimal conditions were in place. The only practicing ophthalmologist in town had an office suite that was well staffed and fully equipped for laser treatment. $\mathrm{He}$ offered his office as the site for screening clinics and conducted screening examinations along with office visits from patients in his regular caseload.

Another small community with one community hospital and one diabetes educator had no ophthalmologist. However, an ophthalmologist from a larger community approximately 100 miles away expressed willingness to participate in the screening project. He had laser treatment equipment in his office.

In a third small community, two ophthalmologists were approached to determine their interest in participating in screening clinics. Neither ophthalmologist had access to laser equipment. Both declined to participate and were very clear about their reasons for this decision. Providing ophthalmologic evaluations at no charge for many people in the community with diabetes would reduce their regular caseload, and any patients detected with significant retinopathy would have to be referred to another ophthalmologist in another community for treatment. Although council members cited the benefits of visibility, community service, and the potential for new patient recruitment for routine ophthalmologic care and treatment of conditions not requiring laser therapy, both ophthalmologists were firm in their decision not to participate.

In one large community with several hospitals and ophthalmologists with laser capabilities in their offices, the council had no difficulty in recruiting a volunteer. However, to alleviate the concern of some ophthalmologists that the volunteer would "get all the referrals," a list of all ophthalmologists in the city and the hospital(s) at which they practiced was distributed to all patients.

Education of people with diabetes and their health care providers is integral to resolving the problem of vision loss from diabetic eye disease. Community-based screening clinics provide an opportunity for local health professionals to detect diabetic eye disease and uncover other potential health risks in the diabetic population. Messages about accepted ophthalmic evaluation guidelines can also be effectively delivered to patients while disseminating the same guidelines to their physicians during clinic publicity and in follow-up. Patient awareness of available diabetes education resources and other hospital services is also fostered in this setting. This screening model affords the opportunity for diabetes educators and other interested health professionals to provide community service, education related to eye care, and information about other important topics in diabetes management.

The authors wish to thank the following diabetes educators for their assistance in the organization and operation of the community retinopathy screening clinics: Sue K. Dennis, Inservice Director, Huron Memorial Hospital, Bad Axe; Mary DesOrmeau. RN, CDE, Outpatient Diabetes Education Coordinator, Alpena General Hospital; Susan L. McIntyre, RN, CDE, Diabetes Educator, Blodgett Memorial Medical Center, Grand Rapids.

\section{References}

1. The Diabetic Retinopathy Study Research Group. Report no. 8: Photocoagulation treatment of proliferative diabetic retinopathy: clinical applications of diabetic retinopathy study finding. Ophthalmology 1981;88:583.

2. National Diabetes Advisory Board. The treatment and control of diabetes: a national plan to reduce mortality and morbidity. Atlanta: Centers for Disease Control, 1981; DHEW publication no. 81-2284.

3. National Diabetes Advisory Board. The prevention and treatment of five complications of diabetes-a guide for primary care practitioners. Atlanta: Centers for Disease Control, 1983; DHHS publication no. 83-8392.

4. Diabetic retinopathy guidelines. Lansing: Michigan Department of Public Health, 1984.

5. Stross JK, Harlan WR. The dissemination of new medical information. JAMA 1979;241:2622-24.

6. Centers for Disease Control. Improving eye care for persons with diabetes. Mich MMWR 1985;34:697-99.

7. Hiss RG, ed. Diabetes in communities. Ann Arbor: The University of Michigan Diabetes Research and Training Center, 1986.

8. Klein R, Klein BEK, Moss SE, Davis MD, DeMets DL. The Wisconsin Epidemiologic Study of Diabetic Retinopathy. II. Prevalence and risk of diabetic retinopathy when age at diagnosis is less than 30 years. Arch Ophthalmol 1984;102:520-26.

9. Klein R, Klein BEK, Moss SE, Davis MD, DeMets DL. The Wisconsin Epidemiologic Study of Diabetic Retinopathy. III. Prevalence and risk of diabetic retinopathy when age of diagnosis is 30 or more years. Arch Ophthalmol 1984;102:527-32.

10. Maliszewski M, Dennis C. DeCoste KC. Prevention, detection and treatment of diabetic eye disease: an overview and demonstration project. Diabetes Educ 1988; 14:416-20. 